\title{
BMJ Open Effects of temperature, humidity, air quality and anthropic activities on the transmission of SARS-CoV-2: a systematic review protocol
}

\author{
Chandrika Jeevananthan (D) , ${ }^{1,2}$ Nor Asiah Muhamad (D) ,2 Mohd Hasni Jaafar (D) , \\ Rozita Hod (D) , ${ }^{1}$ Rimah Melati Ab Ghani, ${ }^{2}$ Zaleha Md Isa (D) , ${ }^{1}$ On behalf of Review \\ Team Members
}

To cite: Jeevananthan $\mathrm{C}$, Muhamad NA, Jaafar MH, et al. Effects of temperature, humidity, air quality and anthropic activities on the transmission of SARS-CoV-2: a systematic review protocol. BMJ Open 2020;10:e039623. doi:10.1136/ bmjopen-2020-039623

- Prepublication history and supplemental material for this paper is available online. To view these files, please visit the journal online (http://dx.doi. org/10.1136/bmjopen-2020039623).

Received 22 April 2020

Revised 11 September 2020

Accepted 04 October 2020

Check for updates

(c) Author(s) (or their employer(s)) 2020. Re-use permitted under CC BY-NC. No commercial re-use. See rights and permissions. Published by BMJ.

${ }^{1}$ Department of Community Health, Faculty of Medicine, Universiti Kebangsaan Malaysia, Kuala Lumpur, Malaysia ${ }^{2}$ Sector for Evidence-based Healthcare,National Institutes of Health, Ministry of Health, Shah Alam, Selangor, Malaysia

Correspondence to Dr Chandrika Jeevananthan; chandrika.jeevananthan@gmail. com

\section{ABSTRACT}

Introduction The current global pandemic of the virus that emerged from Hubei province in China has caused coronavirus disease in 2019 (COVID-19), which has affected a total number of 900036 people globally, involving 206 countries and resulted in a cumulative of 45693 deaths worldwide as of 3 April 2020. The mode of transmission is identified through airdrops from patients' body fluids such as during sneezing, coughing and talking. However, the relative importance of environmental effects in the transmission of the virus has not been vastly studied. In addition, the role of temperature and humidity in air-borne transmission of infection is presently still unclear. This study aims to identify the effect of temperature, humidity and air quality in the transmission of SARS-COV-2.

Methods and analysis We will systematically conduct a comprehensive literature search using various databases including PubMed, EMBASE, Scopus, CENTRAL and Google Scholar to identify potential studies. The search will be performed for any eligible articles from the earliest published articles up to latest available studies in 2020 . We will include all the observational studies such as cohort case-control and cross-sectional studies that explains or measures the effects of temperature and/or humidity and/or air quality and/or anthropic activities that is associated with SARSCoV-2. Study selection and reporting will follow the Preferred Reporting Items for Systematic Reviews and Meta-Analyses (PRISMA) guidelines and Meta-Analysis of Observational Studies in Epidemiology guideline. All data will be extracted using a standardised data extraction form and quality of the studies will be assessed using the Newcastle-Ottawa Scale guideline. Descriptive and meta-analysis will be performed using a random effect model in Review Manager File.

Ethics and dissemination No primary data will be collected, and thus no formal ethical approval is required. The results will be disseminated through a peer-reviewed publication and conference presentation.

PROSPERO registration number CRD42020176756.

\section{INTRODUCTION}

The recent global pandemic of the virus that emerged from Hubei province in China has caused coronavirus disease in 2019 (COVID19), which has affected a total number of
Strengths and limitations of this study

- This review specifically will include studies on transmission of SARS-CoV-2 particularly related to temperature and humidity.

- This review will specifically include studies on COVID-19 confirmed cases with association to air temperature and/or humidity in the study demographic areas.

- This review will systematically retrieve and summarise all observational studies adhering to the standard guidelines for data collection and reporting of findings.

- This review will be the first of its kind to include meta-analysis to provide evidence and increase the precision of quantitative estimates of exposures related to the transmission of SARS-CoV-2.

900036 people globally, involving 206 countries and resulted in a cumulative of 45693 deaths worldwide as of 3 April 2020. ${ }^{1}$ The Coronaviridae Study Group of the International Committee on Taxonomy of Viruses has established that the virus is genetically related to the coronavirus responsible for the SARS outbreak in 2003 which lead to the official naming of SARS-CoV-2 on 11 February $2020{ }^{2-4}$ This virus has caused a negative impact to healthcare services and economic crisis of the affected countries. ${ }^{5}$ SARS-CoV-2 is presumed to be spread by droplets in close prolonged unprotected human to human exposure $;^{6-8}$ however, the relative importance of environmental effects in the transmission of the virus has not vastly studied. In addition, the role of temperature, humidity, air quality and anthropic activities in air-borne transmission of infection is presently still unclear.

Why is it important to do this review?

SARS-CoV-2 has caused a turmoil in the healthcare systems worldwide and within weeks of its outbreak WHO declared it to 
be a global pandemic resulting in closures of international borders subsequently exhausting the economy of affected countries. In a matter of weeks, the world has seen a surge in new cases and steady increase in mortality rates. ${ }^{9}{ }^{10}$ Currently, most studies pertaining to SARS-CoV-2 are vigorously investigating the clinical manifestations and pharmacological aspects of the disease. On the other hand, it is known that the environment plays an important role in the transmission of infectious diseases ${ }^{11}{ }^{12}$ that have not been emphasised. To the present knowledge, very few papers have discussed on the effect of temperature, humidity, air quality and anthropic activities on the transmission of SARS-CoV-2. We believe a comprehensive systematic review will provide the best available evidence on transmission of SARS-CoV-2 and its associated environmental risk factors, particularly evidence on effects of temperature, humidity, air quality and anthropic activities. This review is essential for public health authorities to conduct environmental health assessment, to plan activities for environmental surveillance and control.

\section{Objectives}

To identify the effects of temperature, humidity and air quality in the transmission of SARS-CoV-2 and to determine if the effects are linked to anthropic activities.

\section{Review questions}

For patients who are diagnosed and confirmed COVID19 , we will identify their exposure(s) if any as:

1. Are there any effects on the transmission of SARSCoV-2 to variation of temperature, humidity, air quality and other transmission factors such as drainage systems, indoor and outdoor surface contamination?

2. Is there any association in the variation of temperature or between temperate, tropical and subtropical climate with the incidence of confirmed cases of COVID-19?

3. Is there any association between air quality with indoor and outdoor transmission of SARS-CoV-2?

4. Is there any association between anthropic activities with transmission of SARS-CoV-2?

\section{METHODS}

We will conduct a systematic review in accordance with the Preferred Reporting Items for Systematic Reviews and Meta-Analyses. ${ }^{13}$ As this systematic review will rigorously review observational study designs with meta-analysis as the outcome, we will integrate our reporting with the Meta-Analysis of Observational Studies in Epidemiology guideline. $^{14}$

\section{Patient and public involvement}

Systematic review studies do not require patients and/or public involvement.

\section{Eligibility criteria}

\section{Types of studies}

This systematic review will include all published either peer reviewed, preprint or expedited published articles of observational study designs including cohort, casecontrol, cross-sectional, control cohort before and after (CChBA) and interrupted time series (ITS) that evaluate the risk of exposure for transmission of SARS-CoV-2. We will also include case reports, sentinel surveillance and outbreak reports, which describe on the risk factors associated with transmission of SARS-CoV-2. We will also include guidelines, opinion papers and communication that show potential evidence associated with temperature, humidity and SARS-CoV-2.

\section{Population}

We will include studies that recruit participants at large, as defined by the study authors. We will group the population as similar if they come from the same climate zone.

\section{Types of exposures}

We will include all types of climate conditions as exposures associated with transmission of SARS-CoV-2 among the population. Climate conditions are considered as the variation of temperature, humidity and air quality. Anthropic activities are any human activities that affect the environment such as drainage systems, indoor and outdoor surface contamination which may contribute to the transmission of the SARS-CoV-2. The exposures are defined as (1) measures or variation of estimates of temperature or any environments with moderate rainfall spread across the year or portion of the year with sporadic drought, mild to warm summers and cool to cold winters, ${ }^{15}$ (2) measures of humidity which refers to the presence of water vapour in the air ${ }^{16}$ and (3) measures of anthropic activities. The exposures will be measured as per definition by the study authors or by using validated standardised measurement scales such as sol-air thermometer ${ }^{17}$ and microelectromechanical system. ${ }^{18}$

\section{Types of outcome measures}

The outcomes of this review are divided into primary and secondary outcomes. Primary outcome is total number of confirmed cases of COVID-19 of any severity involving children and adults irrespective of sex and comorbidities as defined in accordance with the standard case definitions by WHO and Centers for Disease Control and Prevention guidelines. ${ }^{19}$ Confirmed case is defined as secondary outcomes are (1) total number of cases in critical care, (2) total number of cases with mild symptoms as defined by study authors or WHO, (3) total number of asymptomatic cases (if available) and (4) total number of deaths. Percentage of cases associated to seasonality and optimum temperature and humidity associated with the highest and the lowest cases will also be identified. 


\section{Information sources}

\section{Electronic searches}

We will systematically conduct a comprehensive literature search using various databases including PubMed, EMBASE, Scopus, CENTRAL and Google Scholar to identify potential studies. We will retrieve earliest eligible published articles up to latest available studies in 2020 . We will adapt the search strategy with the key elements in research question: population, exposure and outcome. We will use a comprehensive search strategy include searching using the Medical Subject Heading (MeSH) terms and free-text terms in the title and abstract on databases (see online supplemental appendix 1). This review will identify potential studies without language restrictions and we will translate the non-English languages abstracts and articles for inclusion in this review. We will also check reference lists from all reviews related to the inclusion criteria.

\section{Selection of studies}

We will employ the standard Cochrane methods, as described in the Cochrane Handbook for Systematic Reviews of Interventions. ${ }^{20}$ Two review authors (CJ and NAM) will independently screen for potentially eligible studies by inspecting the titles and abstracts to generate a shortlist. We record and import search results of their findings in Mendeley-Reference Management Software. Two review authors (CJ and RMAG) then will independently inspect the abstracts or full texts, or both, of these shortlisted studies further to determine final eligibility, using the predefined inclusion and exclusion criteria. We will resolve any disagreement with the help of a third review author (NAM) who acted as an arbiter. We will include published and unpublished studies available in the full-text article or abstract form. Two review authors (MHJ and ZMI) will independently extract data from included study using a dedicated data collection form. We will collect study characteristics such as study design, setting, country, participants, exposure, comparator (if any), outcomes and sponsorship details. We will resolve any discrepancies through discussion with a third author (RH). We will describe the study selection process in a PRISMA diagram. ${ }^{13}$ If multiple reports of the same study are found, we will group them under a single study ID, and assigned the report with the most amount of relevant information as the primary publication. We will summarise all excluded studies after full-text assessment and their reason for exclusion in a 'characteristics of excluded studies' table.

\section{Data extraction}

We will collect study characteristics, including study design, setting, country, participants, exposures, comparators, outcomes, sponsorship details, declaration of interests of the primary investigators and methods used to control possible conflicts of interests, and other information considered relevant according to the Cochrane Handbook for Systematic Reviews of Interventions. ${ }^{20}$
We will resolve potential discrepancies through discussion and by involving a third review author if necessary. Reviewers will use a standardised data extraction form that will be piloted in this review. Two authors will extract the study characteristics, quantitative data (findings on the exposures) and outcome data from included studies.

\section{Risk of bias}

All included papers will be evaluated for study quality. All selected studies will be examined for methodological quality using the Newcastle-Ottawa Scale (NOS) in non-randomised studies of intervention. ${ }^{21}$ The NOS composed of all these aspects: selection, comparability and exposure or outcome. The maximum score is 9 points, where studies with NOS Score $<3$ is classified poor, NOS Score between 3 and $<7$ considered moderate and NOS Scores $\geq 7$ are classified high quality. ${ }^{21}$ Two authors will independently assess the risk of bias for each study according to the criteria incorporated by NOS. We will include studies with potential high risk of bias. We will judge each potential source of bias as high, low or unclear and provide a quote from the study report together with justifications for our judgement in the risk of bias in included studies table. We will grade the level of certainty of evidence with very low, low, moderate and high.

\section{Summary of findings table}

We will create a 'summary of findings' table by tabulating the following outcomes: (1) temperature and (2) humidity. We will assess the quality of evidence for all outcomes using the Grading of Recommendations Assessment, Development and Evaluation (GRADE) methodology. ${ }^{22}$ The GRADE elements consist of study limitations, consistency of effect, indirectness, imprecision and publication bias.

\section{Data analysis and statistical analysis}

We will conduct meta-analysis if there are at least two similar studies with broadly similar population, exposure and outcome measures using a random effect model in Review Manager File. ${ }^{23}$ Studies are considered sufficiently similar if the relevant outcome data are available in the group originally allocated or if the exposures such as temperature in the allocated group belongs to the same category, for example, low temperature during winter, spring and fall or high temperature during summer. We will also categorise the studies as sufficiently similar according to the climates or total rain fall from where the study is conducted. Our primary data analysis will include proportions, prevalence or counts on the positive cases of SARS-CoV-2.

\section{Strategy for data synthesis}

We will consider studies to be sufficiently similar namely with the same category or population, exposure and outcome as defined above. We will conduct a metaanalysis if more than one study that provides usable data in any single comparison is available. We will stratify the analyses in forest plots and tables by type of studies such 
as case-control, cohort or cross sectional, CChBA and ITS. We will chart and tabulate descriptive summary of the results on environmental exposures as defined above. We will describe skewed data and report as medians and IQRs. Data will be qualitatively synthesised based on types of exposures and outcomes.

\section{Assessment of reporting biases}

Publication bias will be assessed by the Bregg's rank correlation and Egger's weighted regression methods ${ }^{24}$ whenever available and we will further create and examine the funnel plots for publication bias.

\section{Reaching conclusions}

We will base our conclusions only on findings from the quantitative or narrative synthesis of included studies for this review. The conclusions can be used as guidelines for top managers and stakeholders in the control and mitigation of the disease. Our implications for research will suggest priorities for future research and outline the remaining uncertainties in the area.

\section{ETHICS AND DISSEMINATION}

We will register this systematic review with the National Medical Research Register, Ministry of Health Malaysia. This protocol will be registered with PROSPERO. We will present the findings of this review in National and International Conferences, Continuous Medical Education and we will disseminate to policy-makers and top managements (stakeholders) of Ministry of Health Malaysia.

Acknowledgements We would like to thank the director general of Health Malaysia for allowing us to publish this systematic review protocol. We would also like to express our gratitude to the National University of Malaysia and National Institutes of Health Malaysia for their support and technical guidance in developing this protocol.

Collaborators Review team members and their organisational affiliations: $\mathrm{Dr}$ Chandrika Jeevananthan (CJ). Department of Community Health, Faculty of Medicine, Universiti Kebangsaan Malaysia; p102415@siswa.ukm.edu.my; Dr Nor Asiah Muhamad (NAM). Sector for Evidence Based Healthcare, National Institutes of Health, Ministry of Health Malaysia; norasiahdr@gmail.com; Associate Professor Dr Mohd Hasni Jaafar (MHJ). Department of Community Health, Faculty of Medicine, Universiti Kebangsaan Malaysia; drmhasni1965@gmail.com; Associate Professor Dr Rozita Hod (RH). Department of Community Health, Faculty of Medicine, Universiti Kebangsaan Malaysia; rozita.hod@ppukm.ukm.edu.my; Dr Rimah Melati Ab Ghani (RMAG). Sector for Evidence Based Healthcare, National Institutes of Health, Ministry of Health Malaysia; rimahmelati@gmail.com; Professor Dr. Zaleha Md Isa (ZMI). Department of Community Health, Faculty of Medicine, Universiti Kebangsaan Malaysia; zms@ppukm.ukm.edu.my.

Contributors CJ and NAM: designed the protocol. CJ, NAM and ZMI: coordinated the protocol. CJ and RMAG: designed search strategies. CJ, MHJ, ZMI, RH, NAM, RMAG: wrote the protocol. NAM, RH and MHJ: provided general advice on the protocol and acted as the guarantor of the protocol.

Funding The authors have not declared a specific grant for this research from any funding agency in the public, commercial or not-for-profit sectors.

Competing interests None declared.

Patient consent for publication Not required.

Provenance and peer review Not commissioned; externally peer reviewed.

Supplemental material This content has been supplied by the author(s). It has not been vetted by BMJ Publishing Group Limited (BMJ) and may not have been peer-reviewed. Any opinions or recommendations discussed are solely those of the author(s) and are not endorsed by BMJ. BMJ disclaims all liability and responsibility arising from any reliance placed on the content. Where the content includes any translated material, BMJ does not warrant the accuracy and reliability of the translations (including but not limited to local regulations, clinical guidelines, terminology, drug names and drug dosages), and is not responsible for any error and/or omissions arising from translation and adaptation or otherwise.

Open access This is an open access article distributed in accordance with the Creative Commons Attribution Non Commercial (CC BY-NC 4.0) license, which permits others to distribute, remix, adapt, build upon this work non-commercially, and license their derivative works on different terms, provided the original work is properly cited, appropriate credit is given, any changes made indicated, and the use is non-commercial. See: http://creativecommons.org/licenses/by-nc/4.0/.

\section{ORCID iDs}

Chandrika Jeevananthan http://orcid.org/0000-0003-1161-0720

Nor Asiah Muhamad http://orcid.org/0000-0002-7772-2103

Mohd Hasni Jaafar http://orcid.org/0000-0003-0007-6008

Rozita Hod http://orcid.org/0000-0001-8645-1723

Zaleha Md Isa http://orcid.org/0000-0003-4850-901X

\section{REFERENCES}

1 WHO. Coronavirus disease 2019 (COVID-19) outbreak situation. Available: https://www.who.int/emergencies/diseases/novelcoronavirus-2019

2 Coronaviridae Study Group of the International Committee on Taxonomy of Viruses. The species severe acute respiratory syndrome-related coronavirus: classifying 2019-nCoV and naming it SARS-CoV-2. Nat Microbiol 2020;5:536-44.

3 Walker PJ, Siddell SG, Lefkowitz EJ, et al. Changes to virus taxonomy and the International Code of virus classification and nomenclature ratified by the International Committee on taxonomy of viruses (2019). Arch Virol 2019;164:2417-29.

4 van Doremalen N, Bushmaker T, Morris DH, et al. Aerosol and surface stability of SARS-CoV-2 as compared with SARS-CoV-1. N Engl J Med NEJM 2020:2004973.

5 Jackson JK, Weiss MA, Schwarzenberg AB. Congressional research service. Global economic effects of COVID-19. Available: https://fas. org/sgp/crs/row/R46270.pdf

6 Bourouiba L. Turbulent gas clouds and respiratory pathogen emissions: potential implications for reducing transmission of COVID-19. JAMA 2020;323:1837-8.

7 Lai C-C, Shih T-P, Ko W-C, et al. Severe acute respiratory syndrome coronavirus 2 (SARS-CoV-2) and coronavirus disease-2019 (COVID-19): the epidemic and the challenges. Int J Antimicrob Agents 2020;55:105924.

8 Yuen K-S, Ye Z-W, Fung S-Y, et al. SARS-CoV-2 and COVID-19: the most important research questions. Cell Biosci 2020;10:40.

9 Chan KW, Wong VT, Tang SCW. COVID-19: an update on the epidemiological, clinical, preventive and therapeutic evidence and guidelines of integrative Chinese-Western medicine for the management of 2019 novel coronavirus disease. Am J Chin Med 2020:1-26.

10 Weiss P, Murdoch DR. Clinical course and mortality risk of severe COVID-19. Lancet 2020;395:1014-5.

11 World Health Organization. Special programme for research and Trainig in tropical diseases. Dis Topic Environ 2019.

12 Semenza JC, Sudre B, Oni T, et al. Linking environmental drivers to infectious diseases: the European environment and epidemiology network. PLoS Negl Trop Dis 2013;7:e2323.

13 Moher D, Liberati A, et al, The PRISMA Group. Preferred reporting items for systematic reviews and meta-analyses: the PRISMA statement. BMJ 2009;2009:b2535.

14 Stroup DF, Berlin JA, Morton SC, et al. Meta-analysis of observational studies in epidemiology: a proposal for reporting. JAMA 2000;283:2008-12.

15 Simmons M. Climates and Microclimates: challenges for extensive green roof design in hot climates. Green roof ecosystems. Springer, Cham 2015:63-80.

16 Pielke RA, Cenedese C, Enfield DB. Climate. meteorology. Encyclopædia Britannica 2019;27.

17 Olofsson T, Ohlsson KEA, Östin R. Measurement of the environmental temperature using the sol-air thermometer. Energy Procedia 2017;132:357-62.

18 Liu Z, Cheng H, Luo Z, et al. Performance assessment of a humidity measurement system and its use to evaluate moisture characteristics 
of wheelchair cushions at the user-seat interface. Sensors 2017:17:775.

19 World Health Organization. Coronavirus disease 2019 (COVID-19) situation Report-63 highlights, 2020. Available: https://www.who.int/ docs/default-source/coronaviruse/situation-reports/20200323-sitrep63-covid-19.pdf?sfvrsn=b617302d_4

20 Higgins JPT, Thomas J, Chandler J, et al. Cochrane handbook for systematic reviews of interventions. 2 nd edn. Chichester, UK: John Wiley \& Sons, 2019.

21 Wells GA, Shea BJ, O'Connell J. The Newcastle-Ottawa scale (NOS) for assessing the quality of nonrandomized studies in meta- analysis. Ottawa Health Research Institute, 2014. http://www.ohri.ca/ programs/clinical_epidemiology/oxford.asp

22 Guyatt G, Oxman AD, AkI EA, et al. Grade guidelines: 1. IntroductionGRADE evidence profiles and summary of findings tables. J Clin Epidemiol 2011;64:383-94.

23 The Nordic Cochrane Centre The Cochrane Collaboration. RevMan 5.3 user quide, 2014. Available: https://training.cochrane.org/sites/ training.cochrane.org/files/public/uploads/resources/downloadable resources/English/RevMan_5.3_User_Guide.pdf

24 Egger M, Smith GD, Phillips AN. Meta-Analysis: principles and procedures. BMJ 1997;315:1533-7. 